\title{
MÉTODOS DE OBTENÇÃO E APLICAÇÕES SINTÉTICAS DE TIAZÓIS, UMA IMPORTANTE CLASSE DE COMPOSTOS HETEROCÍCLICOS
}

Marcus Vinícius Nora de Souza*, Sabrina B. Ferreira, Jorge Souza Mendonça, Marília Costa e Fabiane Ramos Rebello

Fundação Oswaldo Cruz, Farmanguinhos, Rua Sizenando Nabuco, 100, 21041-250 Rio de Janeiro - RJ

Recebido em 12/11/03; aceito em 25/5/04; publicado na web em 9/9/04

\begin{abstract}
METHODOLOGIES FOR THE PREPARATION AND SYNTHETIC APPLICATION OF THIAZOLES, AN IMPORTANT CLASS OF HETEROCYCLIC COMPOUNDS. Thiazoles comprise an important class of heterocyclic compounds present in many potent biologically active molecules. This heterocyclic ring also shows various applications in organic synthesis to obtain new compounds. This paper presents different methodologies for the preparation of thiazole ring systems, as well as their synthetic applications.
\end{abstract}

Keywords: thiazole; biological activity; synthesis.

\section{INTRODUÇÃO}

Os compostos tiazolínicos (Figura 1) são uma importante classe de compostos heterocíclicos que apresentam um amplo espectro de atividade biológica, tais como atividades anticâncer ${ }^{1}$, antibacteriana ${ }^{2}$, antidepressiva $^{3}$, antiinflamatória ${ }^{4}$, herbicida ${ }^{5}$ dentre outras. O núcleo tiazolínico está presente, também, como subunidade em diversas moléculas biologicamente ativas. Como exemplo, podemos mencionar o sulfatiazol ${ }^{6}$, um importante fármaco antimicrobiano, o ritonavir $\left(\right.$ Norvir $\left.^{\circledR}\right)$ medicamento utilizado no combate ao vírus $\mathrm{HIV}^{7}$, as epotilonas A e B, produtos naturais que possuem uma potente atividade antitumoral ${ }^{8}$ e o pirofosfato de tiamina ${ }^{9}$, presente em uma variedade de enzimas que catalisam reações de descarboxilação (descarboxilases) e de condensação do tipo aldólica (aldolases) (Figura 2). Esta classe de compostos apresenta, também, potencial aplicação em síntese orgânica, podendo ser utilizada em reações de condensação, oxidação, transformação de grupos funcionais e formação de ligação carbono-carbono ${ }^{10}$. O presente trabalho tem como objetivo apresentar diferentes metodologias para a obtenção de núcleos tiazolínicos, bem como suas aplicações em síntese orgânica.<smiles>C[SH]1C=CN=C1</smiles>

Figura 1. Núcleo tiazolínico

\section{PREPARAÇÃO DE TIOAMIDAS E TIOURÉIAS}

Devido à presença de compostos tiocarbonilados como tioamidas, tiouréias, isocianatos, etc., em diferentes metodologias para a preparação de núcleos tiazolínicos, faz-se necessário, primeiramente abordar a preparação de compostos tiocarbonilados, que pode ser realizada basicamente por dois reagentes: o primeiro, consiste na utilização do pentasulfeto de fósforo $\left(\mathrm{P}_{4} \mathrm{~S}_{10}\right)^{11}$ (Esquema 1). No entanto, este método apresenta diversas desvantagens, já que requer excesso de $\mathrm{P}_{4} \mathrm{~S}_{10}$, ambiente inerte, solvente anidro (dioxano ou tetrahidrofurano), um tempo prolongado de reação e baixos rendimentos. Um segundo reagente, que pode ser empregado de maneira muito mais

*e-mail: marcos_souza@far.fiocruz.br

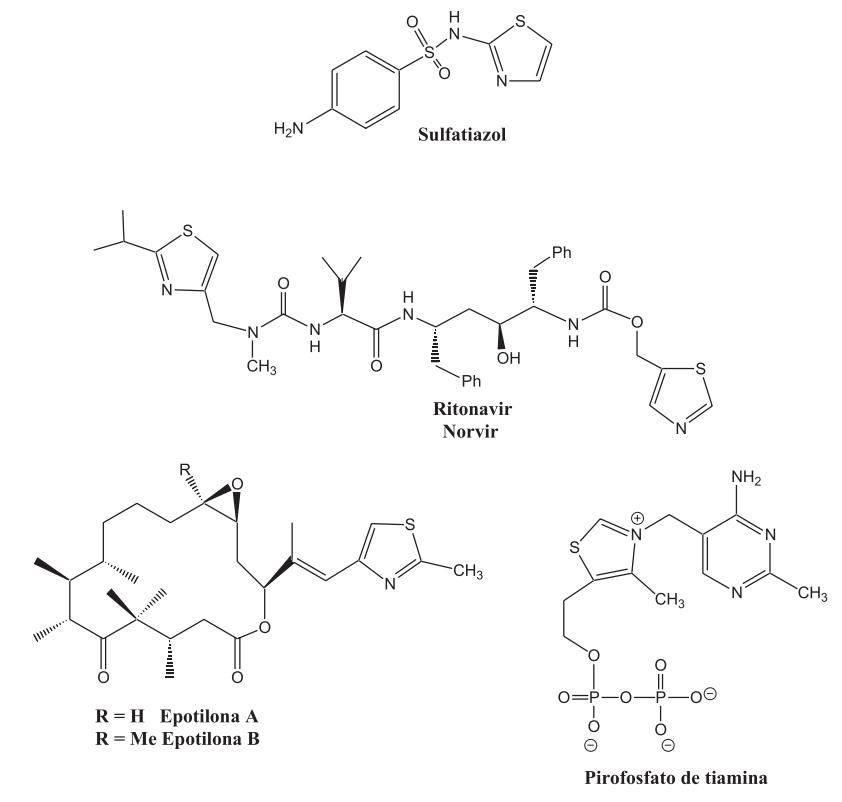

Figura 2. Importantes moléculas bioativas que possuem núcleo tiazolínico

eficaz que o pentasulfeto de fósforo na obtenção de compostos tiocarbonilados a partir de compostos carbonilados, consiste na utilização do 1,3-ditio-2,4-difosfatano-2,4-disulfeto-2,4-bis(4-metóxifenil), comumente conhecido como reagente de Lawesson ${ }^{12}$ (Esquema 1). Este reagente permite uma reação eficaz, limpa, de fácil purificação, realizada em condições brandas e com excelentes rendimentos (Esquema 1).

O mecanismo desta reação é baseado na formação de um intermediário chave 3, que é similar ao da reação de Witting para formação de olefinas a partir de aldeídos e cetonas (Esquema 2).

\section{MÉTODOS DE OBTENÇÃO DE TIAZÓIS}

\section{Síntese de núcleos tiazolínicos a partir de compostos} $\alpha$-halocarbonílicos

Esta metodologia, desenvolvida por Hantzsch em $1887^{13}$, é baseada na condensação de compostos $\alpha$-halocarbonílicos com 


$$
\begin{array}{ll}
\text { a } \mathrm{R}=\mathrm{Ph} & \mathrm{R}^{1}=\mathrm{R}^{2}=\mathrm{CH}_{3} \\
\text { b } \mathrm{R}=\mathrm{Bn} & \mathrm{R}^{1}=\mathrm{R}^{2}=\mathrm{CH}_{3} \\
\text { c } \mathrm{R}=\mathrm{Ph} & \mathrm{R}^{1}=\mathrm{R}^{2}=\mathrm{CH}_{3} \\
\text { d } \mathrm{R}=\mathrm{Bn} & \mathrm{R}^{1}=\mathrm{R}^{2}=\mathrm{CH}_{3} \\
\text { e } \mathrm{R}=\mathrm{Ph} & \mathrm{R}^{1}=\mathrm{H} \mathrm{R}^{2}=\mathrm{CH}_{3} \\
\text { f } \mathrm{R}=\mathrm{Bn} & \mathrm{R}^{1}=\mathrm{HR}^{2}=\mathrm{CH}_{3}
\end{array}
$$

a) Método Clássico: THF, refluxo, excesso de $\mathrm{P}_{4} \mathrm{~S}_{10}$, tempo prolongado (40-70\%) b) Reagente de Lawesson, THF, t.a. (80-95\%)

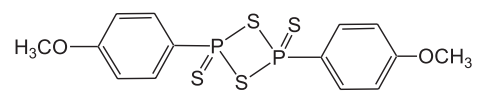

Reagente de Lawesson

Esquema 1. Diferentes metodologias para obtenção de tioamidas

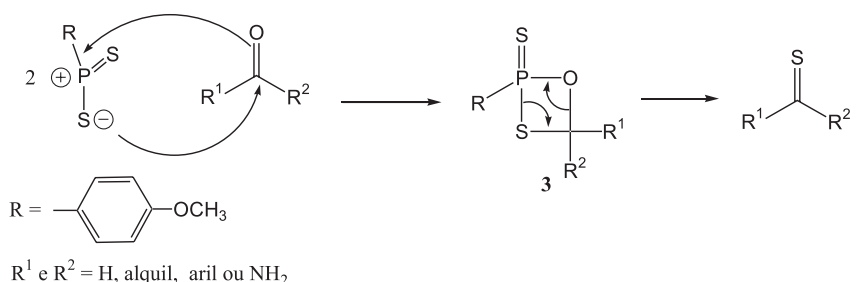

Esquema 2. Proposta mecanística da reação de Lawesson

tioamidas ou tiouréias substituídas ou não, sendo um dos primeiros e mais amplos métodos sintéticos empregados para obtenção de tiazóis substituídos ${ }^{13}$. Como exemplo, apresentamos a obtenção de tiazóis4-amino condensados $\mathbf{6}$, que podem ser facilmente preparados, utilizando $\alpha$-bromolactamas $\mathbf{4}$ e tioamidas $\mathbf{5}^{14}$, sendo a reação aplicada com $\alpha$-bromolactamas de seis, sete e oito membros (Esquema 3 ).

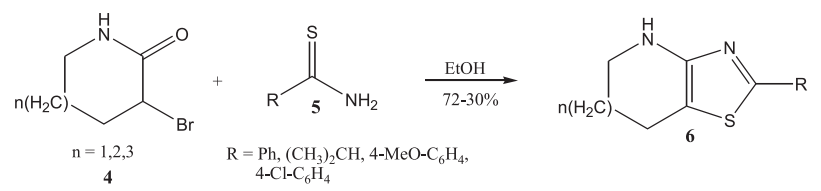

Esquema 3. Preparação de tiazóis 4 amino condensados, utilizado-se $\alpha$ bromolactamas e tioamidas via metodologia de Hantzsch

O mecanismo da reação de $\alpha$-lactamas halogenadas 4 e tioamidas $\mathbf{5}^{14}$ (Esquema 4) pode ser explicado primeiramente pelo ataque nucleofílico do composto 5 sobre a bromo lactama 4, fornecendo assim, o intermediário 7. Após esta etapa, ocorre a ciclização, que produz o intermediário 8 , podendo a formação do produto $\mathbf{6}$ ocorrer por dois caminhos mecanísticos diferentes: o primeiro é a aromatização via desidratação em meio ácido 9, o segundo ocorre pela abertura do biciclo $\mathbf{8}$, seguido de um ataque nucleofílico do etanol sobre a carbonila do intermediário $\mathbf{1 0}$ com posterior desidratação, produzindo o intermediário 11. Após substituição nucleofílica aromática, obtêm-se os respectivos tiazóis 6 (Esquema 4).

Um outro exemplo importante da utilização da metodologia de Hantzsch é a síntese de pirazoiltiazóis $\mathbf{1 5}$, realizada por Bonacorso e colaboradores $^{15}$, a partir de tiocarboxiamidas substituídas $\mathbf{1 2}$ e diferentes 2-bromocetonas 13. Após desidratação em meio ácido do intermediário 14, os pirazoiltiazóis foram obtidos em bons rendimentos (Esquema 5).

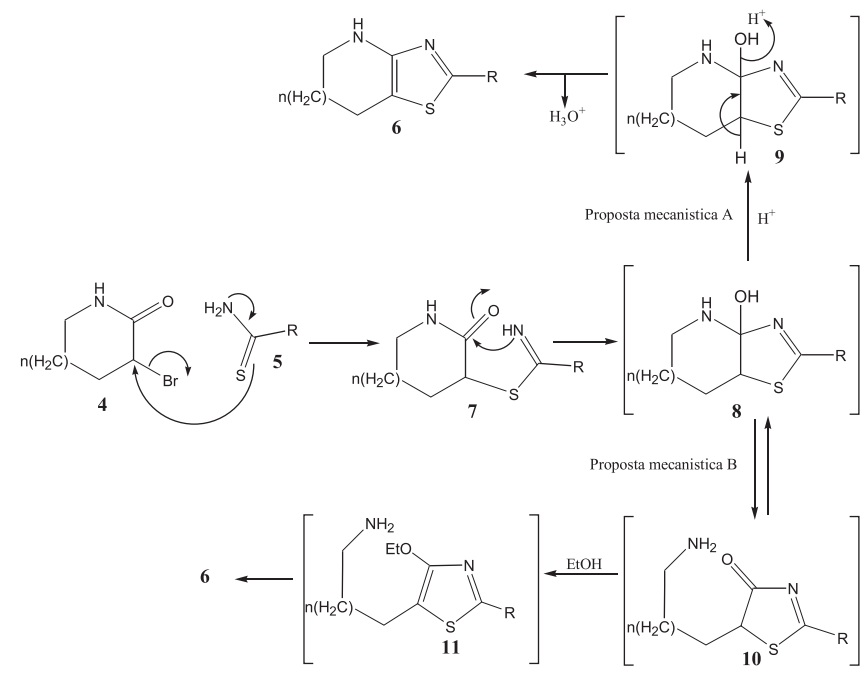

Esquema 4. Proposta mecanística para a formação de tiazóis 4 amino condensados, utilizado-se $\alpha$-bromolactamas e tioamidas
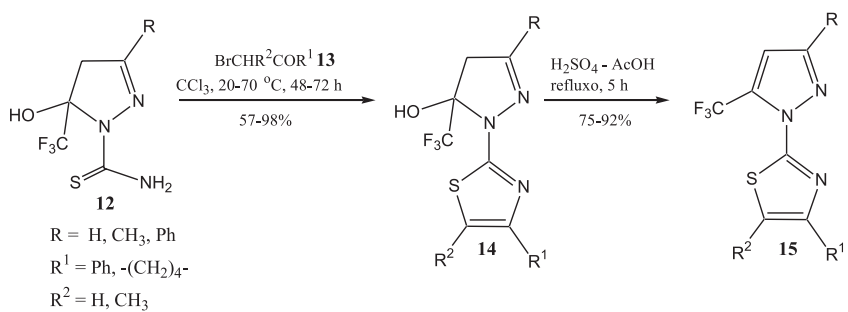

$\mathrm{R}^{2}=\mathrm{H}, \mathrm{CH}_{3}$

Esquema 5. Síntese de pirazoiltiazóis a partir de tiocarboxamidas substituídas e diferentes 2-bromocetonas

\section{Preparação de núcleos tiazolínicos hidroxilados na posição C-4}

A reação de tioamidas 16 e $\alpha$-haloacilas 17 é um método importante para a obtenção de tiazóis hidroxilados 18 na posição C-4 (Esquema 6$)^{16}$. Se a reação é conduzida em presença de cloreto de terbutildimetilsilila (TBDMSCl), obtêm-se em uma etapa (“one-pot”) tiazóis hidroxisililados 19a-e na posição C-4 com rendimentos entre 43 e $78 \%$ (Esquema 6)

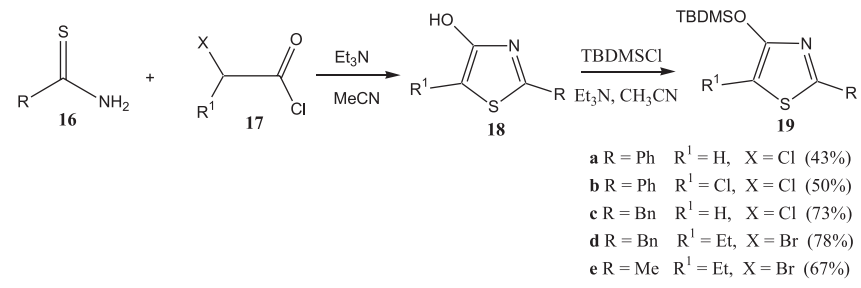

Esquema 6. Obtenção "one-pot" de 4-t-butilsililoxitiazolas a partir de tioamidas e $\alpha$-haloacilas

\section{Síntese de 5-carboxilatos-2-aminotiazóis}

Um método eficiente de preparação de 5-carboxilatos-2aminotiazóis 23a-e foi realizado por Zhao e colaboradores ${ }^{17}$. Esse método consiste na preparação in situ do $\alpha$-haloéster 21, a partir do $\beta$-etóxiacrilato de etila 20 em presença de $N$-bromosuccinimida (NBS), seguido da condensação com diferentes tiouréias 22a-e, fornecendo assim, 5-carboxilatos-2-aminotiazóis $N$-substituídos 23a-e em bons rendimentos (60-98\%) (Esquema 7). 


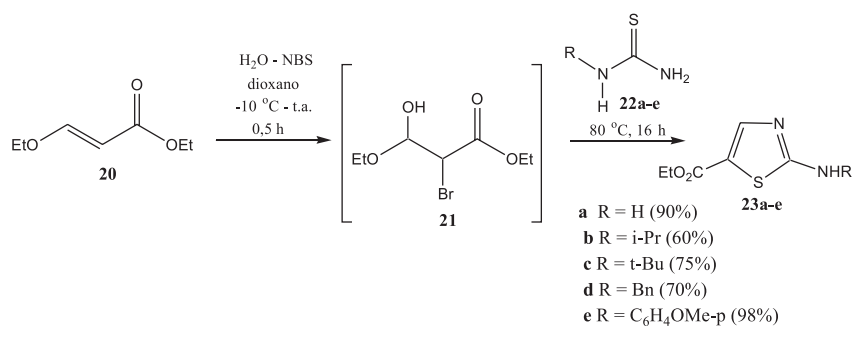

Esquema 7. Preparação "one-pot" de 5-carboxilatos-2-aminotiazóis $N$ substituídos a partir do $\beta$-etóxiacrilato de etila

\section{Iodos hipervalentes}

A utilização de reagentes contendo iodos hipervalentes é uma modificação da síntese de Hantzsch (Esquema 3), que consiste no tratamento de compostos $\beta$-dicarbonilados 24 e 25 com hidroxi(tosiloxi)iodo benzeno e tiouréia, fornecendo 5-carboximetil4-metil-2-aminotiazóis 26 e 27 em 77 e 78\%, respectivamente (Esquema 8$)^{18}$. Essa metodologia apresenta as seguintes vantagens em relação à síntese de Hantzsch: não utiliza compostos $\alpha$-halocarbonílicos e a reação é realizada "one-pot".

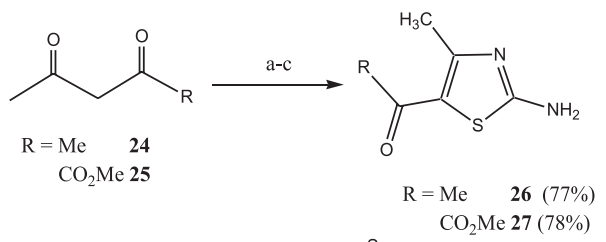

a) $\mathrm{PhI}(\mathrm{OH}) \mathrm{OTs}, \mathrm{CH}_{3} \mathrm{CN}, 45 \mathrm{~min}$; b) ${ }_{\mathrm{H}_{2} \mathrm{~N}} \stackrel{\mathrm{N}}{\mathrm{S}}_{\mathrm{NH}_{2}}$, refluxo, 4 h; c) $\mathrm{NaHCO}_{3}$ (aq.), t.a., 2 h

Esquema 8. Utilização de iodo hipervalente na preparação do 5 carboximetil-4-metil-2-aminotiazóis

\section{Síntese de tiazóis a partir de $\alpha$-aminonitrilas}

A síntese de tiazóis a partir de $\alpha$-aminonitrilas como material de partida é conhecida como Cook-Heilbron ${ }^{19}$. Como exemplo, podese mencionar a preparação de 2-arilamino-4-ciano-5-aminotiazóis 30a-d a partir de $p$-toluenosulfonato de aminomalononitrila 28 e aril isotiocianatos 29a-d, em presença de 1-metil-2-pirolidinona (NMP) como solvente (Esquema 9) ${ }^{20}$.

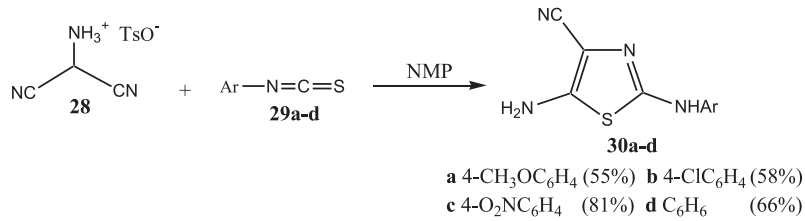

Esquema 9. Preparação de 2-arilamino-4-ciano-5-aminotiazóis a partir de p-toluenosulfonato de aminomalononitrila e de aril isotiocianato

\section{Síntese de 4-ácidos carboxílicos-5-aminotiazóis}

A síntese de 4-carboximetil-5-aminotiazóis 33a-d (62-88\%) ${ }^{21}$ foi realizada por Matsumoto e colaboradores, utilizando $\alpha$ isocianoacetatos $\mathbf{3 1}$ e alquil ou aril isotiocianato 32a-d, em presença de base para a obtenção de núcleos tiazolínicos 4,5-substituídos (Esquema 10).

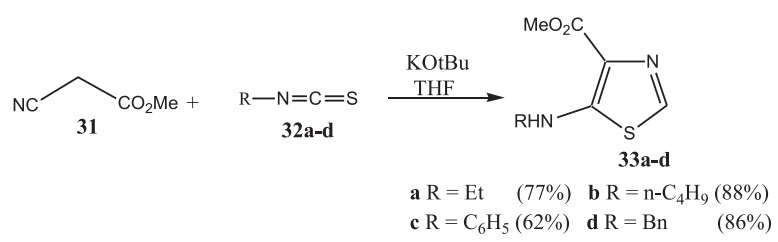

Esquema 10. Síntese de 4-ácidos carboxílicos-5-aminotiazóis a partir de $\alpha$-isocianoacetatos e isotiocianatos em presença de base

\section{Preparação de tiazóis fluorados em posição C-5}

A preparação de núcleos tiazolínicos fluorados em posição C-5 35a-c $(27-69 \%)^{22}$ foi realizada por Burger e colaboradores, utilizando 4,4'-bis(triflúormetil)tiocarboxamidas 34a-c, em presença de cloreto de estanho II $\left(\mathrm{SnCl}_{2}\right)$ (Esquema 11). O mecanismo proposto (Esquema 12) 22 é baseado inicialmente na formação do intermediário cíclico 36 que, após abertura do anel 37, é capaz de doar um átomo de flúor ao cloreto de estanho, obtendo-se o intermediário 38. Após a saída da espécie $\mathrm{SnCl}_{2} \mathrm{~F}$, ocorre a formação do intermediário 39, o qual fornece, após protonação, o produto 40. Após aquecimento, tem-se o intermediário cíclico $\mathbf{4 1}$ que, após a eliminação de HF, fornece os respectivos tiazóis 5-fluorados 35a-c. a) $\mathrm{SnCl}_{2}$, THF, $120{ }^{\circ} \mathrm{C}, 4-24 \mathrm{~h}$

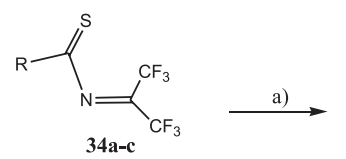

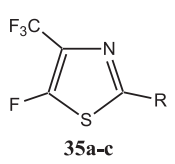

a $\mathrm{R}=\mathrm{Ph}(53 \%) \quad$ b $\mathrm{R}=2-\mathrm{ClC}_{6} \mathrm{H}_{4}(69 \%)$ c $\mathrm{R}=\left(\mathrm{CH}_{3}\right)_{2} \mathrm{~N}(27 \%)$
Esquema 11. Preparação de tiazóis fluorados em posição C-5 utilizandose 4,4'-bis(triflúormetil)tiocarboxamidas em presença de $\mathrm{SnCl}_{2}$

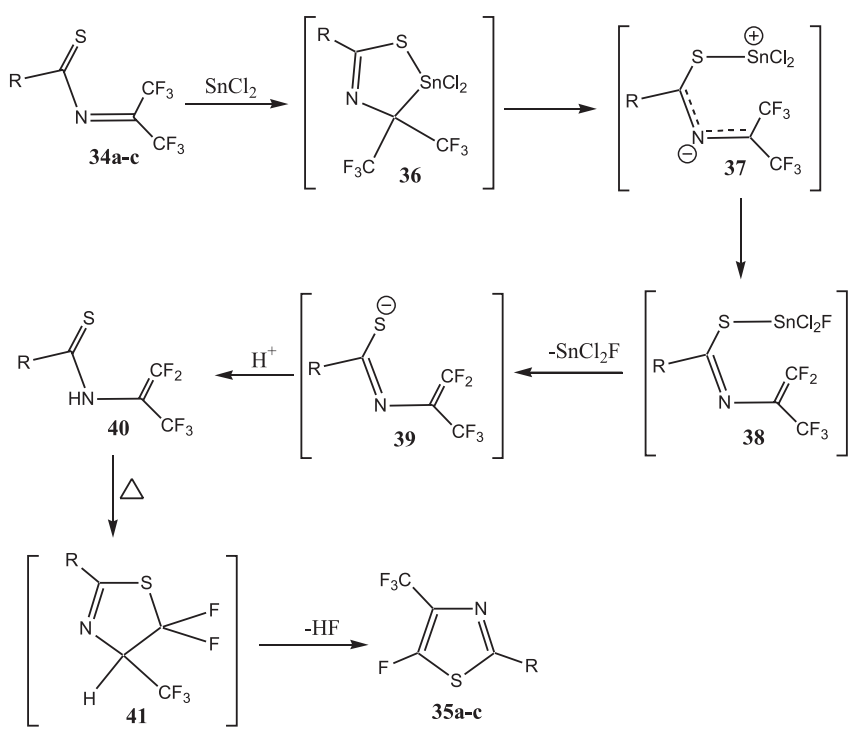

Esquema 12. Proposta mecanística para a preparação de tiazóis fluorados em posição $C-5$

\section{Preparação de 5-tiotiazóis}

A preparação de 5-tiotiazóis 43a-d (21-61\%) $)^{23}$ foi realizada por Junjappa e colaboradores, utilizando como material de partida $S, N-$ acetal cetocetenos $\mathbf{4 2 a - d}$, em presença de cloreto de tionila e piridina (Esquema 13). O mecanismo desta reação (Esquema 14$)^{23}$ é baseado 
na formação do sulfeno 45 que, após abstração de hidrogênio em presença de piridina, fornece o intermediário $\mathbf{4 6}$ que, em seguida, sofre ciclização obtendo-se os correspondentes $S$-óxidos tiazolinas 47. Após a reação do intermediário 47 com cloreto de tionila, obtêm-se os compostos 48, os quais são convertidos em 5-tiotiazóis 43a-d.<smiles>[R]CNC(=CC([R])=O)S[R]</smiles><smiles>[R]Sc1nc([R])sc1C([R])=O</smiles>

a $\mathrm{R}^{1}=\mathrm{C}_{6} \mathrm{H}_{5}, \quad \mathrm{R}^{2}=\mathrm{Me}, \mathrm{R}^{3}=\mathrm{Ph}(60 \%)$

b $\mathrm{R}^{1}=4-\mathrm{CH}_{3} \mathrm{Ph}, \mathrm{R}^{2}=\mathrm{Me}, \mathrm{R}^{3}=\mathrm{Ph}(58 \%)$

c $\mathrm{R}^{1}=4-\mathrm{ClPh}, \quad \mathrm{R}^{2}=\mathrm{Me}, \mathrm{R}^{3}=\mathrm{Ph}(61 \%)$

d $\mathrm{R}^{1}=\mathrm{Me}, \quad \mathrm{R}^{2}=\mathrm{Me}, \mathrm{R}^{3}=\mathrm{Ph}(20 \%)$

Esquema 13. Preparação de 5-tiotiazóis utilizando-se S,N-acetal cetocetenos em presença de cloreto de tionila e piridina

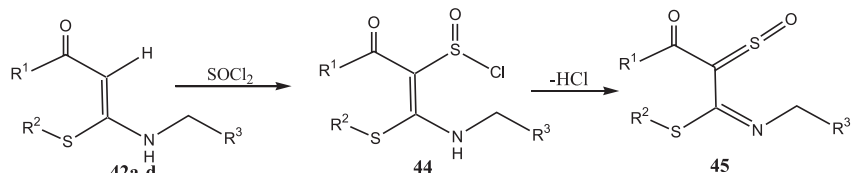
42a-d

44

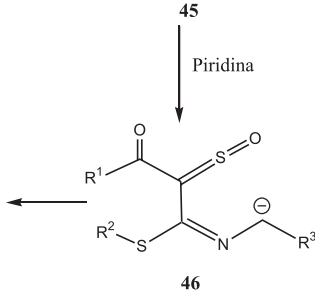

48

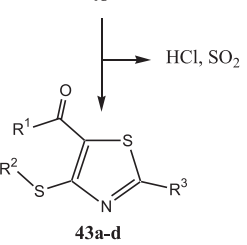

Esquema 14. Proposta mecanística para a preparação de 5-tiotiazóis

\section{Preparação de derivados 2-etóxitiazol}

A preparação do derivado 2-etóxitiazol $51(95 \%)^{24}$ foi realizada por Ziegler e colaboradores, utilizando $N$-acilpropargililtiocarbamatos 49, que são convertidos regioseletivamente em (E)- $\beta$-iodo(vinil) sulfonas 50, por meio de benzenosulfonato de sódio e iodo, em presença de acetato de etila e água (Esquema 15).

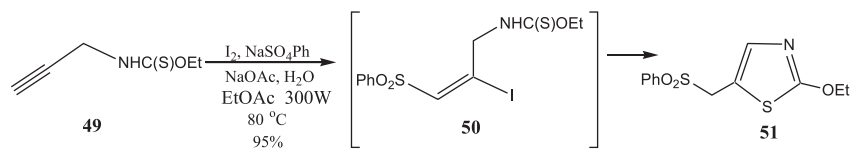

Esquema 15. Preparação de derivados 2-etóxitiazóis a partir de $\mathrm{N}$ acilpropargililtiocarbamatos

\section{Síntese a partir de aminoácidos}

Uma metodologia importante na preparação de núcleos tiazolínicos é a utilização de aminoácidos como material de partida. Como exemplo, pode-se citar a síntese de tiazóis 2,4-dissubstituídos, intermediários chaves 55 e 62 (Esquema 16) ${ }^{25}$ na síntese do produto natural marinho micotiazol, isolado a partir da Spongia mycofijiensis, possuindo potente atividade antielmíntica. Manta e colaboradores realizaram a síntese desse núcleo, utilizando aminoácidos como pro- duto de partida ${ }^{25}$. O primeiro esquema sintético utilizou-se o cloridrato do éster etílico da L-cisteína $\mathbf{5 2}$ como material de partida. Após benzilação do enxofre e acoplamento peptídico com ácido 2,2dimetilacetoacético em presença de DCC (dicicloexilcarbodiimida) e HOBT (1-hidroxibenzotriazol), tem-se o sinton 53. A desproteção do grupo benzila foi realizada em presença de sódio e amônia, seguida da ciclização em presença de $\mathrm{TiCl}_{4}$, fornecendo o intermediário 54 que, após oxidação com $\mathrm{MnO}_{2}$, produziu o tiazol 2,4-dissubstituído

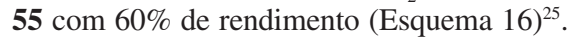

Uma outra proposta sintética realizada por Malta (Esquema 16) ${ }^{25}$, consiste na utilização do cloridrato do éster etílico da L-serina $\mathbf{5 6}$ que, após acoplamento peptídico com ácido 2,2-dimetilacetoacético, em presença de DCC (diciclohexilcarbodiimida) e HOBT (1hidroxibenzotriazol), levou ao intermediário 57. Após a proteção do álcool 57 com TBDMSCl foi realizada a transformação da cetona em tiocetona, utilizando o reagente de Lawesson produzindo, assim, o produto 59. Em seguida, foi realizada a desproteção do álcool 59 em presença de TBAF (fluoreto de tetrabutilamônio), fornecendo o intermediário 60. A ciclização deste intermediário é realizada em presença do reagente de Burgess-PEG, obtendo-se o composto $6 \mathbf{1 .}$ Finalmente, tem-se a oxidação deste sinton, fornecendo o tiazol 2,4dissubstituído 62 (Esquema 16).

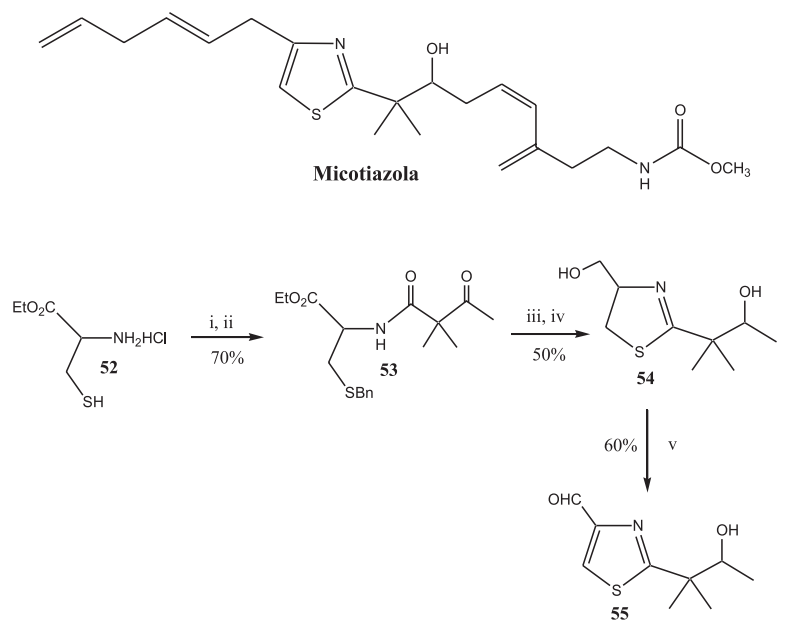

i) $\mathrm{BnCl}, \mathrm{Et}{ }_{3} \mathrm{~N}$, EtOH; ii) ácido 2,2-dimetilacetoacético, DCC, $\mathrm{HOBT}, \mathrm{CH}_{2} \mathrm{Cl}_{2}, 0{ }^{\circ} \mathrm{C}$; iii) $\mathrm{Na}-\mathrm{NH}_{3},-78^{\circ} \mathrm{C}$ iv) $\mathrm{TiCl}_{4}, \mathrm{CH}_{2} \mathrm{Cl}_{2}$, refluxo; v) $\mathrm{MnO}_{2}, \mathrm{CH}_{2} \mathrm{Cl}_{2}$, refluxo

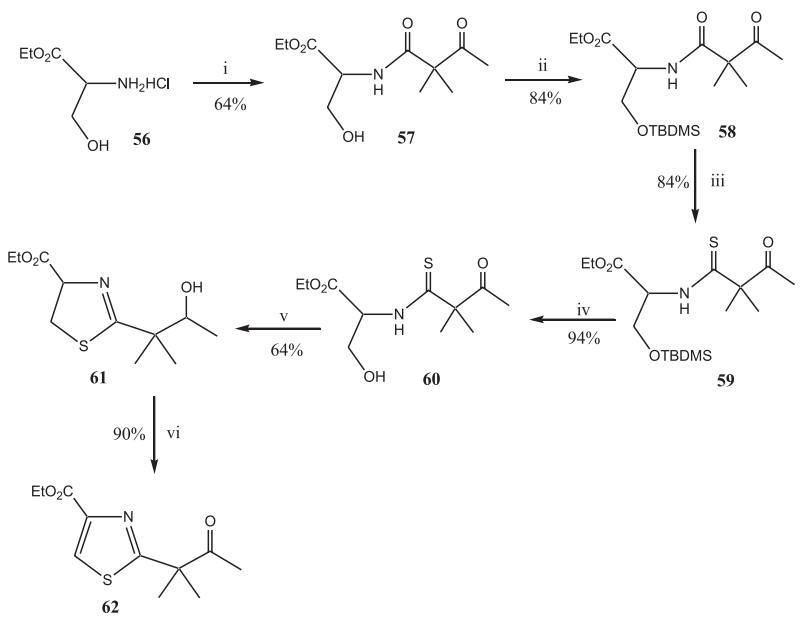

i) ácido 2,2-dimetilacetoacético, DMAP, DCC, $\mathrm{CH}_{2} \mathrm{Cl}_{2},{ }^{\circ} \mathrm{C}$; ii) TBDMSCl, imidazol, DMAP, $\mathrm{CH}_{2} \mathrm{Cl}_{2}$, t.a.; iii) reagente de Lawesson, PhH, refluxo; iv) TBAF, THF, t.a.; v) Burges-PEG, dioxano-THF, $85^{\circ} \mathrm{C}$ vi) $\mathrm{MnO}_{2}, \mathrm{CH}_{2} \mathrm{Cl}_{2}$, refluxo

Esquema 16. Preparação da síntese de tiazóis 2,4-dissubstituídos a partir de aminoácidos, intermediários chaves na síntese do produto natural marinho micotiazol 
A importância da utilização de aminoácidos ${ }^{26}$ na síntese de núcleos tiazolínicos quirais 63a-d pode ser ilustrada pelo planejamento sintético dos peptídeos cíclicos marinhos lissoclinamida $6 \mathrm{e}$ ulitiaciclamida B, elaborado por Pattenden e colaboradores ${ }^{26}$ (Figura 3).

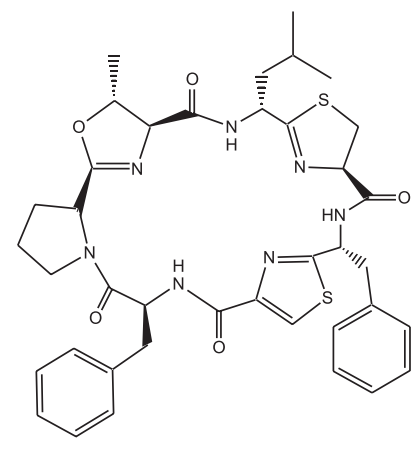

Lissoclinamida 6

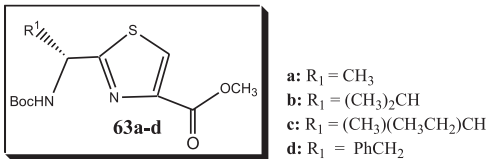

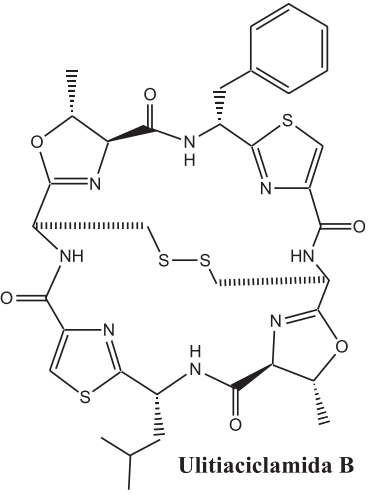

d: $\mathrm{R}_{1}=\mathrm{PhCH}_{2}$
Figura 3. Lissoclinamida 6 e Ulitiaciclamida B, peptídeos cíclicos marinhos

Na construção dos núcleos tiazolínicos quirais 63a-d, (Esquema 17) três rotas sintéticas foram realizadas ${ }^{26}$ : a primeira foi baseada na condensação de aminoácidos $N$-protegidos 64a-d e imino éteres 65a-d, obtendo os intermediários 66a-d (30-65\%), que após oxidação forneceu tiazóis quirais 63a-d (84-88\%). A segunda consiste na ciclodesidratação de $\beta$-hidroxitioamidas 68a-d, usando as condições de Mitsunobu $^{27}$ ou o reagente de Burgess ${ }^{28}$, fornecendo os intermediários 66a-d (40-50\%). Finalmente, o acoplamento de tioamidas 69a-d com bromo piruvato de etila, seguido de desidratação, fornece os respectivos tiazóis quirais 63a-d (35-44\%) (Esquema 17).

Ao comparar as três rotas sintéticas, Pattenden observou que a primeira rota era a melhor, pois as substâncias de partida utilizadas eram de baixo custo. Outra importante vantagem da primeira rota sintética é que os tiazóis quiras são obtidos sem perda do excesso enantiomérico e em alta escala ${ }^{26}$.

\section{APLICAÇõES SINTÉTICAS DE TIAZÓIS}

Além da atividade biológica dos núcleos tiazolínicos, essa classe de compostos heterocíclicos possue, também, importantes aplicações sintéticas, podendo ser empregada em diferentes tipos de reações de condensação, oxidação, transformação de grupos funcionais e formação de ligação carbono-carbono.

\section{Sais tiazólicos como catalisadores}

Sais tiazólicos podem catalisar reações químicas, sendo amplamente estudados. Como exemplo, pode-se mencionar sua utilização catalítica em reações de condensação de aldeídos, sendo importante o "design" do catalisador para a interação entre os aldeídos aromáticos 70 e os sais tiazólicos 71a,b (Esquema 18) ${ }^{29}$. O mecanismo desta reação foi proposto pela primeira vez por Breslow ${ }^{30}$, baseado no acoplamento dos aldeídos aromáticos 70 com os sais tiazólicos 71a,b, formando os compostos dipolares $\mathbf{7 3}$ e $\mathbf{7 4}$ que, em seguida reagem com outra molécula de aldeído 70, fornecendo a benzoína 75 que, após a regeneração do catalisador $\mathbf{7 1 b}$, produz o composto 72 (Esquema 19).

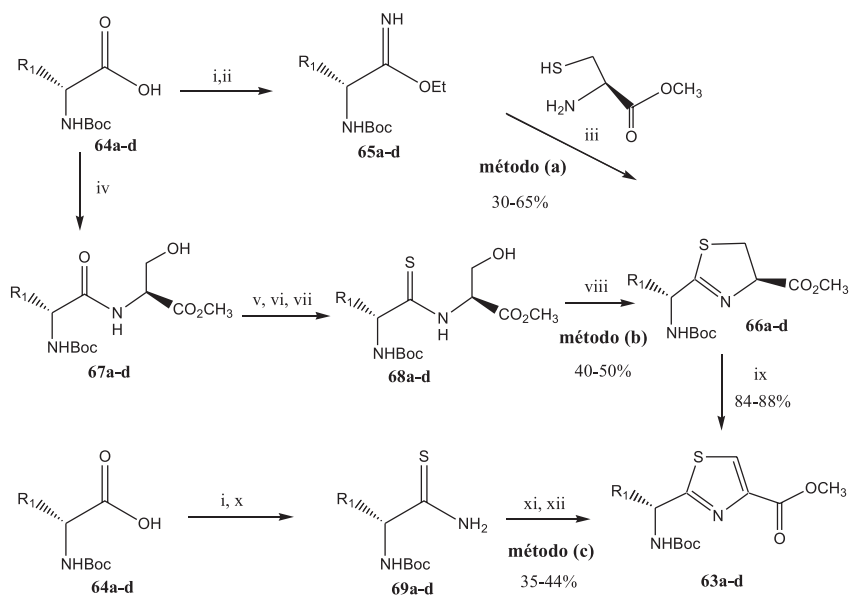

a: $\mathrm{R}_{1}=\mathrm{CH}_{3}$; b: $\mathrm{R}_{1}=\left(\mathrm{CH}_{3}\right)_{2} \mathrm{CH} ; \mathbf{c}: \mathrm{R}_{1}=\left(\mathrm{CH}_{3}\right)\left(\mathrm{CH}_{3} \mathrm{CH}_{2}\right) \mathrm{CH}$; d: $\mathrm{R}_{1}=\mathrm{PhCH}_{2}$

i, EtOCOCl-Et ${ }_{3} \mathrm{~N},-78^{\circ} \mathrm{C} ; \mathrm{NH}_{4} \mathrm{OH},-30^{\circ} \mathrm{C}$; ii, $\mathrm{Et}_{3} \mathrm{O}^{+} \mathrm{PF}_{6}, \mathrm{CH}_{2} \mathrm{Cl}_{2}$; iii, cisteína metilada, EtOH; iv, serina metilada, $\mathrm{HOBt}$, DCC; v, TBSCl, imidazol, DMF; vi, reagente de Lawesson, $\mathrm{PhH}, 80^{\circ} \mathrm{C}$; vii, TBAF, THF; viii, reagente de Burgess (hidróxido de metóxicarbonilsulfamoiltrietilamônio, sal inerte), THF, $65^{\circ} \mathrm{C}$; ix, $\mathrm{MnO}_{2}, \mathrm{CH}_{2} \mathrm{Cl}_{2}$; $\mathrm{x}$, reagente de Lawesson, DME, t.a.; xi, $\mathrm{KHCO}_{3}, \mathrm{DME}, \mathrm{BrCH}_{2} \mathrm{COCO}_{2} \mathrm{Et}$, t.a.; xii, ácido trifluoracético, piridina, DME, $0^{\circ} \mathrm{C}$

Esquema 17. Síntese de núcleos tiazolínicos quirais utilizando aminoácidos como material de partida

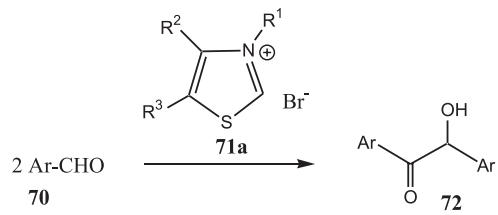

$\mathrm{R}^{1}, \mathrm{R}^{2}$ e $\mathrm{R}^{3}=\mathrm{H}$, alquil, aril

$\mathrm{Ar}=\mathrm{Ph}, p \mathrm{NO}_{2} \mathrm{C}_{6} \mathrm{H}_{5}, p \mathrm{ClC}_{6} \mathrm{H}_{5}$, etc.

Esquema 18. Utilização catalítica de sais tiazólicos em reações de condensação benzoínica

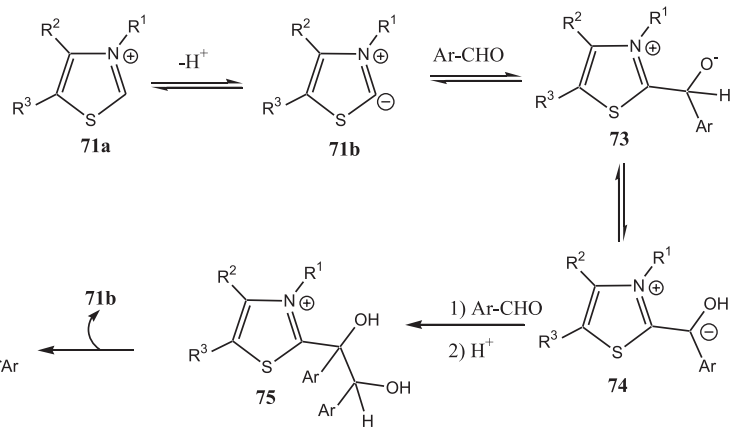

Esquema 19. Proposta mecanística para reações de condensação benzoínica em presença de sais tiazólicos como catalisadores

Uma outra proposta mecanística (Esquema 20) é $^{31}$ baseada na dimerização dos intermediários 71a,b, que forneceu o bis(ilidenotiazolina)s 77, que reage com o aldeído, fornecendo os intermediários em equilíbrio 78 e 79. Após a condensação do intermediário 79 com outra molécula de aldeído, a benzoína $\mathbf{8 0}$ formada produz o aldeído 72, bem como a regeneração do catalisador $\mathbf{7 7}$.

Sais tiazólicos também catalisam diferentes reações relacionadas com a condensação benzoínica. Um exemplo é a reação de aldeídos 81a,b e sais iminiuns 82 em presença do sal tiazólico 83, o qual atua como catalisador na obtenção de $\alpha$-amino cetonas $\mathbf{8 4 a , b}$ (Esquema 21) ${ }^{32}$. 


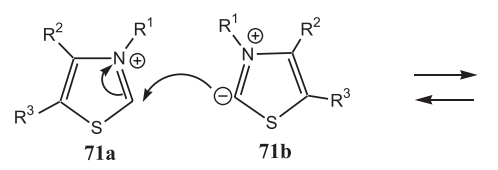
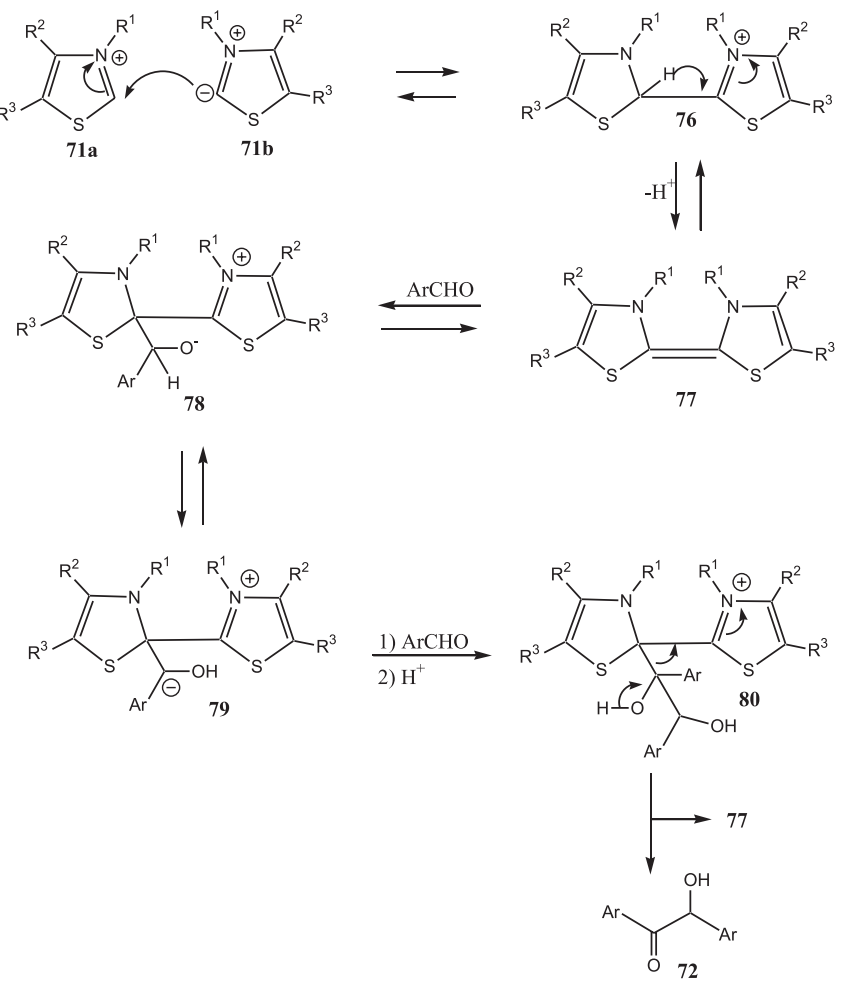

Esquema 20. Proposta mecanística baseada na formação de bis(ilidenotiazolina)s atuando como catalisador em reações de condensação benzoínica

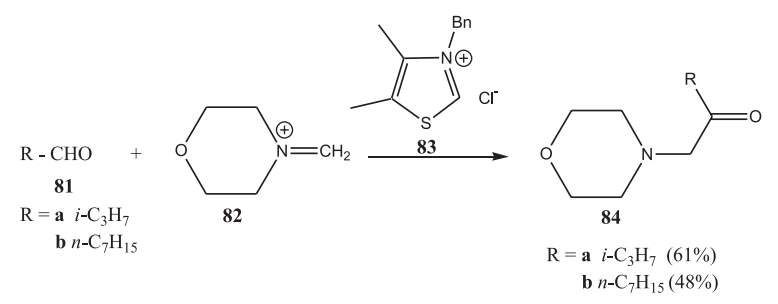

Esquema 21. Reação de aldeídos e sais iminiuns em presença do sal tiazólico 79

A oxidação de aldeídos aromáticos em ácidos carboxílicos $\mathbf{8 8}$, pode também ser catalisada em presença de sais tiazólicos 85 (Esquema 22$)^{33}$. O mecanismo da reação é similar ao apresentado nos Esquemas 19 e 20.

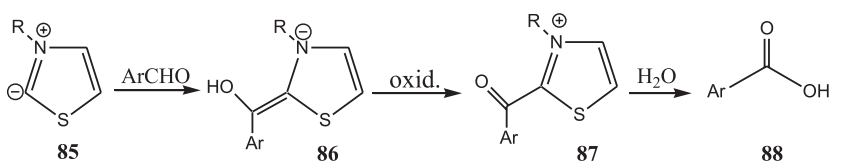

$\mathrm{R}=\mathrm{H}$, alquil ou aril

$\mathrm{Ar}=$ aromático

Esquema 22. Esquema geral de oxidação de aldeídos aromáticos em ácidos carboxílicos catalisados em presença de sais tiazólicos

\section{Preparação de cetonas a partir de ésteres}

A preparação de cetonas a partir de ésteres pode ser facilmente obtida pela utilização de 2-acilbenzotiazóis 91, preparados pela condensação de benzotiazóis litiados 90 em posição 2, e os respectivos ésteres 89 (Esquema 23) (34 $^{34}$ As 2-acilbenzotiazóis 91 formadas reagem com um composto litiado, seguido da formação de um sal em presença de iodeto de metila, obtendo-se o intermediário 92 que, após a utilização de base fornece as respectivas cetonas 94a-c (9497\%) e o sal benzotiazólico 93, que atua como catalisador (Esquema 23).

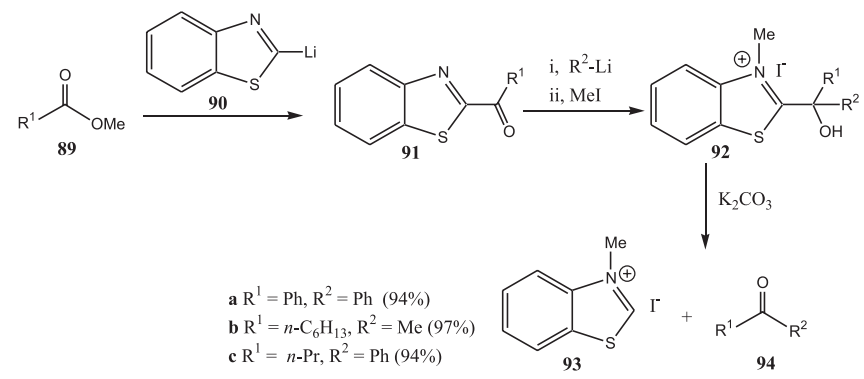

Esquema 23. Preparação de cetonas a partir de ésteres utilizando-se 2acilbenzotiazóis, pela etapa de transformação do núcleo tiazolínico em aldeído

\section{Preparação de 5H-tiazolo[3,2-a] pirimidin-5-onas}

O núcleo tiazolínico foi empregado com sucesso por Bonacorso e colaboradores na síntese de $5 \mathrm{H}$-tiazolo[3,2-a] pirimidin-5-onas $\mathbf{9 7} \mathbf{7 3}^{\mathbf{3 5}}$, utilizando $\beta$-alcoxivinil triclorometil cetonas $\mathbf{9 5}^{36}$, que foram condensadas com 2-amino tiazol fornecendo o bi-heterociclo 97 (Esquema 24). Esta classe de compostos, especialmente o 7-metil-5H-tiazolo [3,2-a] pirimidin-5-onas, apresentou interessante atividade antiúlcera e antiinflamatória após administração oral.

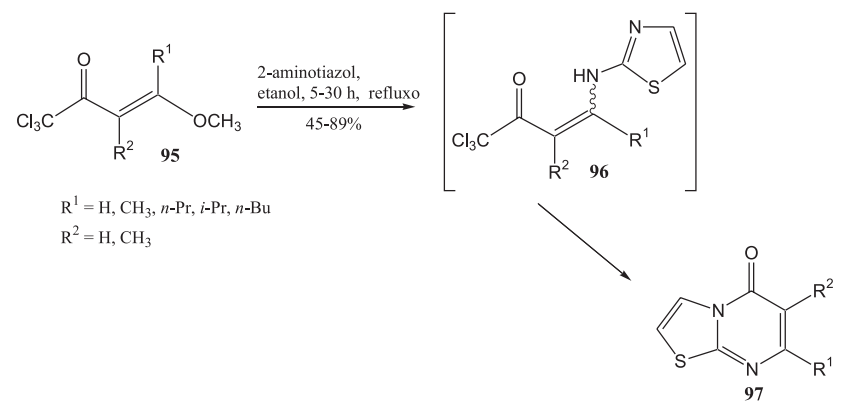

Esquema 24. Preparação de 5H-tiazolo[3,2-a] pirimidin-5-onas

\section{FORMAÇÃO DE LIGAÇÃO C-C}

Uma aplicação sintética importante dos núcleos tiazóis é a sua equivalência com o grupo aldeídico (Esquema 25), contribuindo assim para a formação de ligação carbono-carbono em diferentes sistemas $^{37}$. De maneira geral, o grupo funcional aldeído é introduzido primeiramente pela funcionalização do núcleo tiazolínico em posição C-2 com diferentes grupos funcionais (GF) 98, tais como lítio, os compostos sililados e estanilados, os aldeídos, as cetonas e os reagentes de Witting (Esquema 25). Após a funcionalização dos núcleos tiazolínicos, o substrato R em questão é acoplado originando o composto 99, seguido pela etapa de transformação do núcleo tiazolínico em aldeído (Esquema 25).

Como exemplo, pode-se citar a introdução do grupo funcional aldeído ao serinal $N, N$-diprotegido 100, a qual se inicia com o acoplamento do núcleo tiazolínico, 2-litio tiazol 101, obtendo-se o composto $\sin 102$ em $85 \%$ de rendimento e com excesso enantiomérico (e.e) de $92 \%$, pois a adição ao grupo aldeídico ocorre com seletividade diastereofacial. Após benzilação do álcool formado 103, 


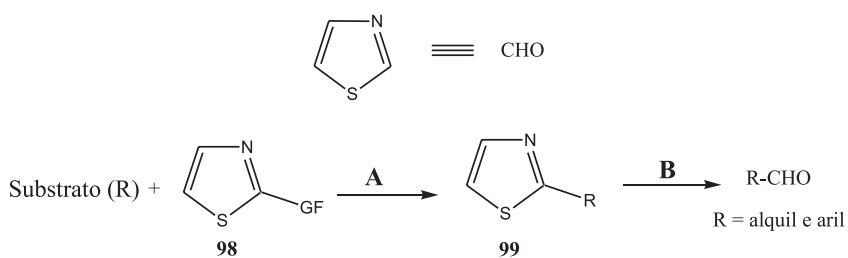

Grupo Funcional $(\mathbf{G F})=\mathrm{Li}, \mathrm{SiMe}_{3}, \mathrm{SnMe}_{3}, \mathrm{CHO}, \mathrm{COMe}, \mathrm{CH}=\mathrm{PPh}_{3}$, etc.

Esquema 25. Equivalência dos núcleos tiazolínicos com o grupo aldeídico e sua aplicação para a formação de ligação carbono-carbono em diferentes sistemas

$N$-metilação do núcleo tiazolínico 96, redução do anel em presença de boroidreto de sódio $\mathbf{1 0 5}$ e, finalmente, utilização do cloreto de mercúrio II, há a obtenção do grupo funcional aldeído 106 com rendimento de 54\% (quatro etapas) ${ }^{38}$ (Esquema 26).
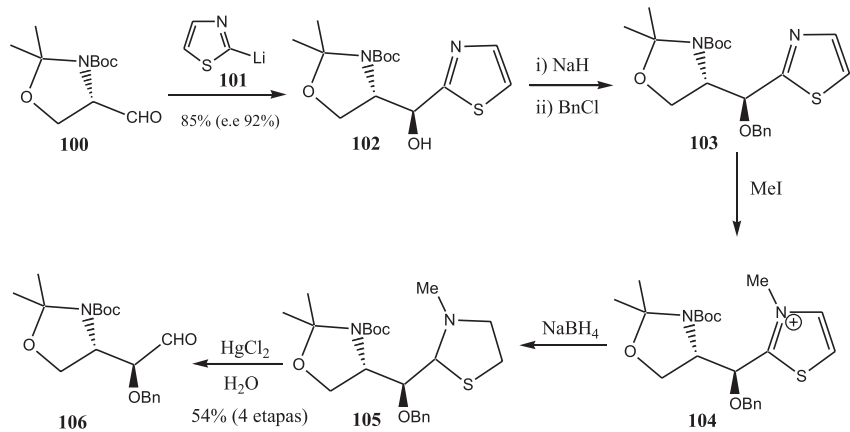

Esquema 26. Síntese de $\alpha$-hidroxialdeídos, utilizando-se tiazóis para formação de ligação $C$ - $C$

Um bom exemplo da importância dos núcleos tiazolínicos na formação de ligação C-C é a síntese total de azaaçúcares ${ }^{39}$ realizada por Dondoni e colaboradores. Esses produtos naturais, como por exemplo a (+)-nojirimicina e (+)-manonojirimicina ${ }^{39}$ (Figura 4) são, respectivamente, potentes inibidores de glicosidase e manosidase. Essa classe de compostos tem sido avaliada como capaz de inibir a glicoproteína viral, essencial para a replicação do vírus HIV. Dondoni e colaboradores realizaram uma elegante síntese assimétrica da (-)-nojirimicina e (-)manonojirimicina, antípodas de seus produtos naturais (Esquema 27) ${ }^{39}$. Essa síntese utiliza a L-serina $\mathbf{1 0 7}$ como material de partida que, após algumas transformações, fornece o sinton 108, ao qual foi introduzido o composto 2-litiotiazol 101, obtendo-se o intermediário chave $109 \mathrm{em}$ $82 \%$ de rendimento. Graças à introdução deste grupo, pode-se obter uma redução esterocontrolada que, dependendo do reagente empregado $\left(\mathrm{NaBH}_{4}\right.$ ou Red-Al), fornece os compostos $\mathbf{1 1 0}$ e $\mathbf{1 1 1}$ com alto excesso enantiomérico (e.e) 94 e 95\%, respectivamente (Esquema 27). Após sililação do álcool secundário, transformação do grupo tiazolínico em aldeído, desproteção do grupo silil em meio ácido e ciclização, obtemse a (-)-nojirimicina e (-)-manonojirimicina em 80 e $72 \%$ respectivamente (Esquema 27) ${ }^{39}$. É importante ressaltar que, a partir da D-serina, se pode obter pelo mesmo processo sintético os produtos naturais (+)nojirimicina e (+)-manonojirimicina.

\section{CONCLUSÃO}

Devido a sua importância, os núcleos tiazolínicos têm sido muito estudados, representando uma importante classe de compostos heterocíclicos, já que estão presentes em diversas substâncias com

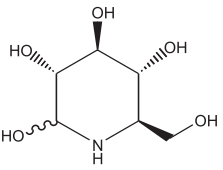

(+)-Nojirimicina

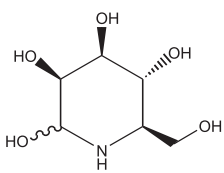

(+)-Manonojirimicina
Figura 4. Produtos naturais (+)-nojirimicina e (+)-manonojirimicina, potentes inibidores de glicosidase e manosidase
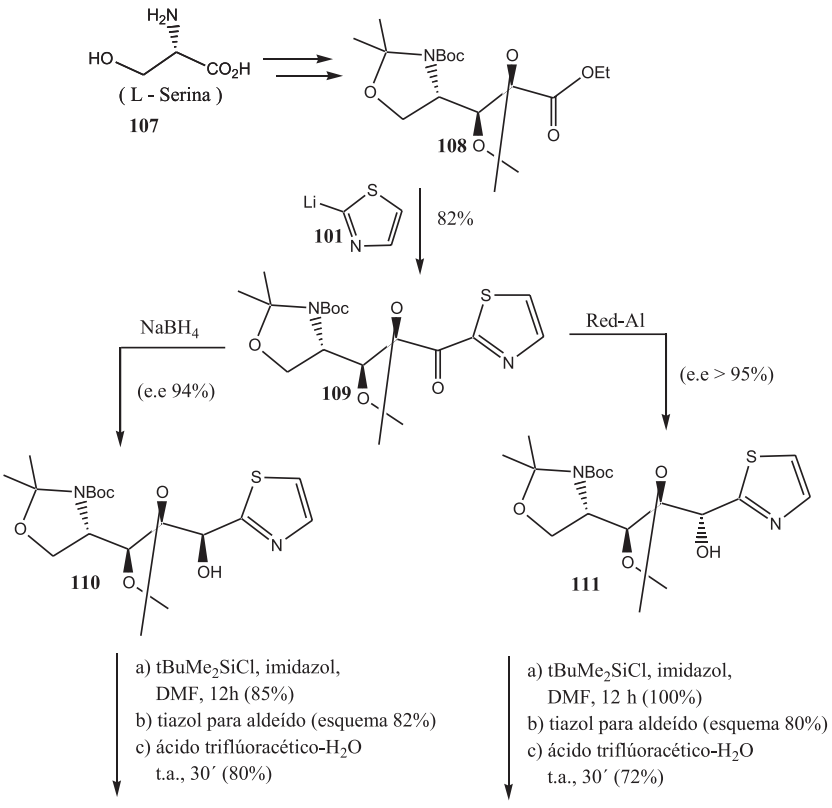

(-) Nojirimicina

(-) Manonojirimicina

Esquema 27. Síntese assimétrica da (-)nojorimicina e (-)manonojirimicina

potente atividade biológica, bem como apresentam diversas aplicações sintéticas para obtenção de novos compostos.

\section{AGRADECIMENTOS}

Os autores agradecem a profa. de português T. H. da Silva pela revisão do manuscrito.

\section{REFERÊNCIAS}

1. Vicini, P.; Geronikaki, A.; Incerti, M.; Busonera, B.; Poni, G.; Cabras, C. A., La Colla, P.; Bioorg. Med. Chem. 2003, 11, 4785.

2. Maccioni, E.; Cardia, M. C.; Bonsignore, L.; Plumitallo, A.; Pellerano, M. L.; De Logu, A.; Il Farmaco 2002, 57, 809; Holla, B. S.; Malini, K. V.; Rao, B. S.; Sarojini, B. F.; Kumari, N. S.; Bioorg. Med. Chem. 2003, 38, 313.

3. Funakoshi, T.; Chaki, S.; Kawahima, N.; Suzuki, Y.; Yoshikawa, R.; Kumagai, T.; Nazakato, A.; Kameo, K.; Goto, M.; Okuyama, S.; Life Sci. 2002, 71, 1371; Harmet, J. J.; Veronique, R.; Dolo, C.; Charnet, C.; Spinnewyn, B.; Cornet, S.; Rolland, A.; Marin, J. G.; Bigg, D.; Chabrier, P. E.; Bioorg. Med. Chem. 2004, 14, 157.

4. Ingelman, S. M.; Simi, A.; Tindberg, N.; WO 0135959, 2001. (CA 134:361399)

5. Laber, B.; Usunow, G.; Wiecko, E.; Franke, W.; Franke, H.; Kohn, A.; Pestic. Biochem. Phyiol. 1999, 63, 173.

6. Kleemann, A.; Engel, J.; Pharmaceutical Substances: Syntheses, Patents and Applications, ${ }^{\text {nd }}$ ed., ThiemeStuttgart: New York, 2001.

7. De Souza, M. V. N.; De Almeida, M. V.; Quim. Nova 2003, 26, 366

8. Kamath, K.; Jordan, M. A.; Cancer Res. 2003, 18, 6026; Storer, R. I.; Takemoto, T.; Jackson, P. S.; Ley, S. V.; Angew. Chem., Int. Ed. 2003, 22, 2521; Nicolau, K.C.; Roschangar, F.; Vourloumis, D.; Angew. Chem., Int. Ed. 1998, 37, 2014; Balog, A.; Meng, D.; Kamenecka, T.; Bertinato, P.; Su, D. S.; Sorenson, E.; Danishefsky, S. J.; Angew. Chem., Int. Ed. 1996, 35, 2801. 
9. Care, D. E.; Science 2000, 287, 818; Svergun, D. I.; Petoukhov, M. V.; Koch, M. H.; Konig, S; J. Biol. Chem. 2000, 287, 818; Leonardi, R.; Fairhurst, S. A.; Kriek, M.; Lowe, D. J.; Roach, P. L.; FEBS Lett. 2003, 539, 95; Park, Y. H.; Dorrestein, P. C.; Zhai, H.; Kinsland, C.; McLafferty, F. W.; Begley, T. P.; Biochemistry 2003, 42,12430. Meenick, K.; Sprinz, K. J.; Reddick, J. J.; Kinsland, C.; Begley, T. P.; Bioorg. Med. Chem. Lett. 2003, 13, 4139.

10. Katritzky, A. R.; Rees, C. W.; Scriven, E. F. V.; Comprehensive Heterocyclic Chemistry II, Elsevier Science: New York, 1996, vol. 3; Katritzky, A. R.; Rees, C. W. Em Comprehensive Heterocyclic Chemistry; Potts, K. T., ed.; 1984, vols. 1-8, Pergamon Press: Oxford.

11. Hurd, R. N.; DeLaMeter, G.; Chem. Rev. 1961, 61, 45.

12. Scheibye, S.; Pedersen, E. S.; Lawesson, S. O.; Bull. Soc. Chim. Belg. 1978, 87, 229; Clausen, K.; Thorsen, M.; Lawesson, S. O.; Tetrahedron 1981, 37, 3635; Lajote, J.; Lepine, F.; Maziak, L.; Belleau, B.; Tetrahedron Lett. 1983, 24, 3815.

13. Hantzsch, A.; Weber, J. H.; Chem. Ber. 1887, 20, 3118; Moreno, I.; Tellitu, I; SanMartin, R.; Badia, D.; Carrilo, L.; Dominguez, E.; Tetrahedron 1999, 40, 5067; Bailey, N.; Dean, A. W.; Judd, D. B.; Middlemiss, J. D.; Storer. R.; Watson, S. P.; Bioorg. Med. Chem. Lett. 1996, 6, 1409; Moody, C. J.; Swann, E.; J. Med. Chem. 1995, 38, 1039; Masharaqui, S. H.; Keehn, P. M.; J. Am. Chem. Soc. 1982, 104, 4461.

14. Uchikawa, O.; Aono, T.; J. Heterocycl. Chem. 1994, 31, 1545; Holla, B. S.; Malini, K. V.; Rao, B. S.; Sarojini, B. K.; Kumari, N. S.; Eur. J. Med. Chem. 2003, 38, 313; Commeureuc, A. G. J.; Murphy, J. A.; Dewis, M. L.; Org. Lett. 2003, 16, 2785; Kazzouli, S. E.; Raboin, S. R.; Mouaddib, A.; Guillaumet, G.; Tetrahedron Lett. 2002, 43, 3193; Cetusic, J. R. P.; Green III, F. R.; Graupner, P. R.; Oliver, M. P.; Org. Lett. 2002, 8, 1307.

15. Bonacorso, H. G.; Wastowski, A. D.; Muniz, M. N.; Zanatta, N.; Martins, M. A. P.; Synthesis 2002, 1079.

16. Kopka, I. E.; Tetrahedron Lett. 1988, 31, 3765.

17. Zhao, R.; Gove, S.; Sundeen, J. E.; Chen, B. C.; Tetrahedron Lett. 2001, 42, 2101; Marcantonio, K. M.; Frey, L. F.; Murry, J. A.; Chen, C.; Tetrahedron Lett. 2002, 43, 8845; Katritzky, A. R.; Wang, X.; Maimait, R.; J. Org. Chem. 2000, 65, 8077.

18. Moriarty, R. M.; Vaid, B. K.; Duncan, M. P.; Stuart, G. L.; Prakash, O.; Goyal, S.; Synthesis 1992, 845

19. Cook, A. H.; Downer, J. D.; Heilbron, I.; J. Chem. Soc. 1948, 2028; Capp, C. W.; Cook, A. H.; Heilbron, I; J. Chem. Soc. 1948, 1340; Cook, A. H.; Heilbron, I.; Smith, E.; J. Chem. Soc. 1949, 1440.

20. Freeman, F.; Darrick, S. H. L. K.; J. Org. Chem. 1991, 56, 4645; Cocco, M. T.; Onnis, V.; Synthesis 1993, 199.

21. Suzuki, M.; Noriya, T.; Matsumoto, K.; Niyoshi, M.; Synthesis 1982, 874; Guy, R. G.; Mountford, P.; Tetrahedron 1987, 28, 117.

22. Burger, K.; Geith, K.; Hubi, D.; Synthesis 1988, 189; Coyanis, E. M.; Della Védora, C. O.; Haas, A.; Merz, A.; J. Mol. Struct. 2003, 660, 147.
23. Rahman, H. I.; Junjappa, H.; Synthesis 1984, 250

24. Short, K. M.; Ziegier, Jr.; Tetrahedron Lett. 1993, 34, 71.

25. Serra, G.; Mahler, G.; Manta, E.; Heterocycles 1998, 48, 2035; Crews, P.; Kakou, Y.; Quinoa E.; J. Am. Chem. Soc. 1988, 110, 4365; Sugiyama, H.; Yokokawa, F.; Shioiri, T.; Tetrahedron 2003, 59, 6579; Sugiyama, H.; Yokokawa, F.; Shioiri, T.; Org. Lett. 2000, 14, 2149.

26. Boden, C. D. J.; Pattenden, G.; Ye T.; Synlett 1995, 417; Jayaprakash, S.; Pattenden, G.; Viljoen, M. S.; Wilson, C.; Tetrahedron 2003, 59, 6637; Arrault, A.; Legrand, A. W.; Gonzalez, F.; Subielos, N. B.; Banaigs, B.; Tetrahedron Lett. 2002, 43, 4041; Clough, J.; Chen, S.; Gordon, E. M.; Hackbarth, C.; Lam, S.; Trias, J.; White, R. J.; Candiani, G.; Donadio, S.; Romano, G.; Ciabatti, R.; Jacobs, J. W.; Bioorg. Med. Chem. Lett. 2003, 13, 3409; De Roy, P. L.; Charette, A. B.; Org. Lett. 2003, 22, 4163.

27. Gaelotti, N.; Montagne, C.; Poncet, J.; Jouin, P.; Tetrahedron Lett. 1992, 33, 2807; Wipf, P.; Miller, C. P.; Tetrahedron Lett. 1992, 33, 6267.

28. Wipf, P.; Fritch, P. C.; Tetrahedron Lett. 1994, 35, 5397.

29. Matsumoto, T.; Ohishi M.; Inoue, S.; J. Org. Chem. 1985, 50, 603; Sheehan, J.C.; Hara, T.; J. Org. Chem. 1974, 39, 1196; Knight, R. L.; Leper, F. J.; J. Chem. Soc., Perkin Trans. 1 1998, 1891.

30. Breslow, R.; J. Am. Chem. Soc. 1957, 79, 1762; Breslow, R.; J. Am. Chem. Soc. 1958, 80, 3719.

31. Castells, J.; Lopez-Calahorra, F.; Domingo, L.; J. Org. Chem. 1988, 53, 4433; Castells, J.; Domingo, L.; Lopez-Calahorra, F.; Martf, J.; Tetrahedron Lett. 1993, 34, 517.

32. Castells, J.; Lopez-Calahorra, F.; Bassedas, M.; Urrios, P.; Synthesis 1988 , 315; Murry, J. A.; Frantz, D. E.; Soheili, A.; Tillyer, R.; Grabowski, E. J, Reider, P. J.; J. Am. Chem. Soc. 2001, 39, 9696.

33. Jimerez, L.; Diederich, F.; Tetrahedron Lett. 1989, 30, 2759.

34. Chikashita, H.; Ishihara, M.; Takigawa, K.; Itoh K.; Bull. Chem. Soc. Jpn. 1991, 64, 3261

35. Bonacorso, H. G.; Wastowski, A. D.; Muniz, M. N.; Zanatta, N.; Martins, M. A. P.; Tetrahedron Lett. 2002, 43, 9315.

36. Siqueira, G. M.; Flores, A. F. C.; Clar, G.; Zanatta, N.; Martins, M. A. P.; Quim. Nova 1994, 17, 24

37. Dondoni, A.; Synthesis 1998, 1681; Patil, N. T.; John, S.; Sabharwal, S. G.; Dhavale, D. D.; Bioorg. Med. Chem. 2002, 10, 2155; Compain, P.; Martin, O. R.; Bioorg. Med. Chem. 2001, 9, 3077; Bordier, A.; Compain, P.; Martin, O. R.; Ikeda, K.; Asano, N.; Tetrahedron: Asymmetry 2003, 14, 47; Koos, M.; Steiner, B.; Micova, J.; Langer, V.; Durek, V.; Gyepesova, D.; Carbohydr. Res. 2001, 332, 351.

38. Dondoni, A.; Fantin, G.; Fogagnolo, M.; Pedrini, P.; J. Org. Chem. 1990 55, 1439; Dondoni, A.; Perrone, D.; Merino, P.; J. Org. Chem. 1995, 60, 8074 .

39. Dondoni, A.; Merino, P.; Perrone, D.; Tetrahedron 1993, 14, 2939. 